\title{
Gasos Guínicos \\ Verruga plantar recalcitrante tratada exitosamente con una sesión de láser PDL
}

\author{
Jaime Pérez ${ }^{1}$, Viviana García ${ }^{2}$, Claudia Telchi ${ }^{3}$, Mariela Gaete ${ }^{1}$
}

\section{RESUMEN}

Las verrugas plantares son una patología común en la población. Estas lesiones son a menudo dolorosas y recidivantes. Entre los tratamientos más utilizados están las terapias tópicas destructivas, y menos frecuentemente, el tratamiento quirúrgico. Sin embargo, con frecuencia recurren, por lo que representan un desafío para los dermatólogos.

Se presenta a un paciente de 29 años con una verruga plantar recalcitrante que fue tratada exitosamente con una sesión de láser PDL, sin recidiva luego de 12 meses de seguimiento.

Palabras claves: verruga plantar; láser; PDL.

\section{SUMmaRY}

Plantar warts are a common pathology in the population. These lesions are often painful and recurring. Among the most used treatments are topical destructive therapies, and less frequently, surgical treatment. However, they frequently recur, so they represent a challenge for the dermatologist.

A 29 years old patient is presented with a recalcitrant plantar wart that was successfully treated with a single PDL laser session, without relapse after 12 months of follow-up.

Key words: plantar warts; laser; PDL.
${ }^{1}$ Servicio de

Dermatología Clínica Alemana de Santiago. Facultad de Medicina Universidad del Desarrollo, Santiago de Chile.

${ }^{2}$ Clínica Indisa, Santiago de Chile. ${ }^{3}$ Becaria 3er año

Dermatología,

Hospital de Clínicas "José de San Martín" Universidad de Buenos Aires, Buenos Aires,

Argentina.

Trabajo no recibió

financiamiento. Los autores declaran no tener conflictos de interés.

Recibido el 30 de mayo de 2019, aceptado el 17 de marzo de 2020.

Correspondencia: Jaime Pérez Email:jjperezw@ yahoo.es
$\mathrm{L}$ as verrugas son tumores benignos causados por la infección por virus papiloma humano en los queratinocitos de piel y mucosas. La incidencia en la población general es del 10\%, de las cuales las verrugas plantares corresponden al $24 \%{ }^{1}$

Los tratamientos más utilizados en la actualidad son la crioterapia, ácido salicílico, 5-Fluoruracilo, bleomicina, imiquimod y escisión quirúrgica; aunque la remisión completa resulta un desafío, ya que normalmente son cuadros de larga evolución y con fracaso terapéutico. ${ }^{1}$

En la actualidad se ha intentado realizar tratamientos láser para las verrugas recalcitrantes, lo que se define como fracaso a los tratamientos tópicos convencionales. $^{2}$

Dentro de los láseres estudiados, se incluyen láser CO2, PDL, Nd-YAG y Er-YAG.

El láser PDL (Pulsed Dye Laser) es el que tiene menos efectos adversos. Se ha postulado que su mecanismo de acción se relaciona con la producción de fototermólisis selectiva sobre los vasos sanguíneos dilatados de las papilas dérmicas de la lesión verrugosa. Además, tendría una acción no selectiva causando daño tisular, lo que activa la respuesta inmune celular y regulación positiva de $\mathrm{IL}^{2-4}$ intralesional, los cuales protegen contra infecciones virales. ${ }^{1}$

El éxito de PDL en verrugas virales varía entre un 48 y $95 \% .^{2}$

Los protocolos de tratamientos sugieren que se realicen sesiones cada 3-4 semanas, según la clínica del paciente, con fluencias de 12, 5 a 15,0 Joule/cm2. ${ }^{2}$

\section{Gaso clínico}

Se presenta paciente de sexo masculino, de 29 años de edad, sin antecedentes patológicos informados, que había consultado en otro centro dermatológico por lesión verrugosa en planta del pie derecho de 1 año de evolución, asintomática. 
Dicha lesión fue tratada con 6 sesiones de nitrógeno líquido, sin mayor respuesta.

Luego consulta en nuestro servicio el 02 de agosto de 2017 para una segunda opinión.

$\mathrm{Al}$ examen físico dermatológico presentaba una lesión verrugosa confluente, en mosaico en la planta del pie derecho, de aproximadamente $3 \mathrm{~cm}$. de diámetro (Figura 1a).

Se indica inicialmente tratamiento con sesiones de nitrógeno líquido. Se completaron en total 11 sesiones de tratamiento con crioterapia.

Por persistencia de lesiones y con diagnóstico de verruga plantar recalcitrante, se indicó tratamiento con láser.

Se decide iniciar terapia con láser Vbeam (PDL), con los siguientes parámetros:

$7,5 \mathrm{~J} / \mathrm{cm} 2-0,45$ y $3 \mathrm{~ms}-7 \mathrm{~mm}-\mathrm{DCD} 60 / 40$.

Se evaluó a paciente clínica y dermatoscópicamente dos semanas después de este tratamiento, observándose escasas costras hemáticas en la periferia, apreciando una mejoría del 100\% por lo que no se realizó nueva sesión. (Figura 1b).

Se mantuvo en control ambulatorio sin recaída luego de un seguimiento de 12 meses (Figura 1c).

\section{Discusión}

Se ha estudiado la terapia con láser para verrugas como terapia de inicio o recalcitrantes a otros tratamientos.

Dentro de éstos se ha estudiado láser CO2, Er:YAG, PDL y Nd: YAG; los cuales presentan tasas de respuesta similares (CO2: 50-100\%, Er YAG: 72-100\%, PDL $47-100 \%$, Nd YAG: 46-100\%). Sin embargo, el PDL es el que ha demostrado menores efectos adversos. ${ }^{1,3}$

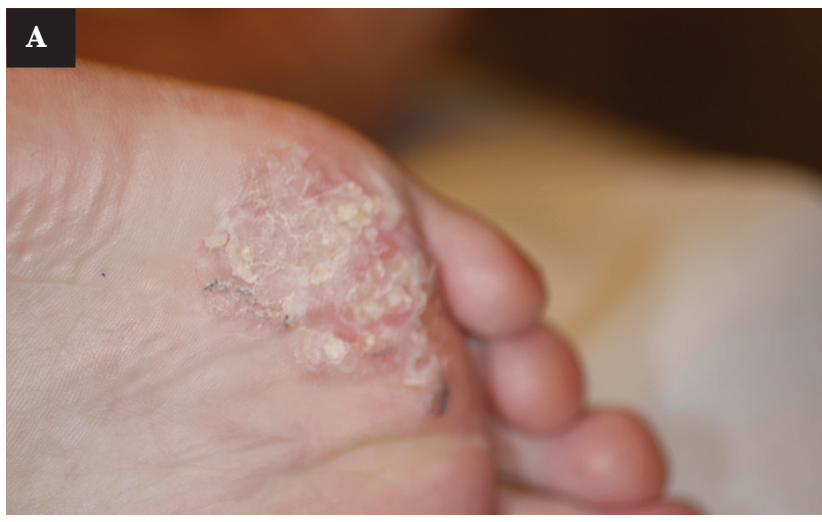

\section{B}

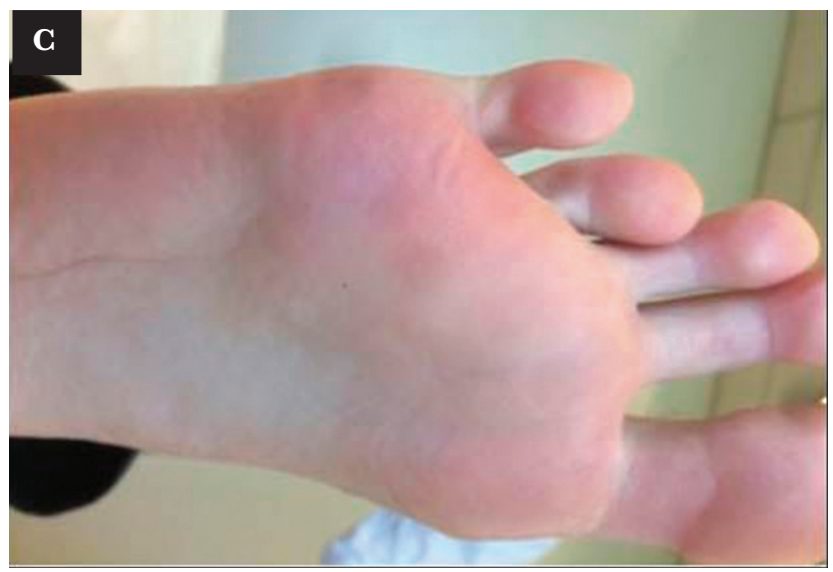

\section{Figura 1}

A. Verruga plantar previa a la sesión de +láser PDL.

B. Control 2 semanas después de 1 sesión de láser PDL.

C. Control 6 semanas después de 1 sesión de láser PDL. 
Tabla 1

Uso de laser PDL en verrugas recalcitrantes

\begin{tabular}{lccccc}
\hline Referencia & N $^{\mathbf{0}}$ Sesiones & Fluencia & Spot & Duración Pulso & Efectividad \\
\hline Sparreboom E et al & $6(\mathrm{x})$ & $12,5-15 \mathrm{~J} / \mathrm{cm} 2$ & $7 \mathrm{~mm}$ & $1,5 \mathrm{~ms}$ & $86 \%$ \\
Shin Y et al & $4(\mathrm{x})$ & $10-14 \mathrm{~J} / \mathrm{cm} 2$ & $7 \mathrm{~mm}$ & $20 \mathrm{~ms}$ & $5,1 \%$ \\
El-Mohamady A et al & $5(\mathrm{x})$ & $8 \mathrm{~J} / \mathrm{cm} 2$ & $7 \mathrm{~mm}$ & $0,5 \mathrm{~ms}$ & $73,9 \%$ \\
Pérez W. Et al & 1 & $7,5 \mathrm{~J} / \mathrm{cm} 2$ & $7 \mathrm{~mm}$ & $0,45-3 \mathrm{~ms}$ & $100 \%$ \\
\hline
\end{tabular}

Por ejemplo, el Láser CO2 presenta efectos adversos tales como: cicatriz e hipopigmentación. Por su parte, con la utilización de láser Er: YAG se ha registrado dolor intraoperatorio y enrojecimiento persistente.

En cuanto al PDL, entre los efectos adversos se incluyen dolor local durante y después del procedimiento, ampollas, costras, cicatrización y cambios transitorios de pigmento, aunque éstos son significativamente menores que con otros láseres y en comparación con la crioterapia. ${ }^{1}$ En un estudio donde se compara Nd:YAG con láser PDL, se observó que Nd:YAG presenta más efectos adversos que PDL, pero con este último se necesitan más sesiones para lograr el mismo resultado. ${ }^{4}$

Se presenta este caso por la excelente respuesta de una verruga plantar recalcitrante con una sola sesión de Láser PDL. El paciente había sido tratado previamente con numerosas sesiones de Nitrógeno líquido sin ninguna respuesta. Si bien, en nuestro servicio el láser es utilizado como una terapia de segunda elección debido a su mayor costo, es opción indicar esta terapia como una primera elección o cambiar de terapia más precozmente, debido a su mayor efectividad. Incluso los costos de una sesión de láser son bastante inferiores a varias sesiones de crioterapia. Sin embargo, la disponibilidad del equipo continúa siendo una limitante. ${ }^{5}$

El láser PDL es equivalente en cuanto a eficacia a terapias convencionales como la crioterapia, con respuesta entre 48 y $95 \% .^{2}$ Esta variación suponemos que depende de los parámetros utilizados los cuales no son fijos y varían según la experiencia del operador. (Tabla 1)

\section{Conalusión}

La terapia con Láser PDL es altamente efectiva y segura para el tratamiento de verrugas plantares y estaría especialmente indicada en casos de verrugas plantares recalcitrantes. Nuestro caso tuvo una mejoría completa con una sola sesión de láser PDL. La decisión de utilizar esta terapia debiera ser más precoz para evitar una dolencia prolongada y el alto costo de numerosas sesiones de crioterapia.

Abreviaturas: PDL: Pulsed Dye Laser; DCD: Dynamic Cooling Device

\section{REFERENCIAS}

1. Nguyen J, Korta D, Chapman L, Kelly K. "Laser Treatment of Nongenital Verrucae: A Systematic Review". JAMA Dermatol. 2016;152(9):1025-34

2. Sparreboom E, Luijks H.G, Luiting-Huelkenhuyzen H.A.L., Willems P.W., et al. "Pulsed-dye laser treatment for recalcitrant viral warts: a retrospective case series of 227 patients". Br J Dermatol. 2014;171(5):1270-3

3. Shin Y, Cho E, Park E, Kim K, et al. "A comparative study of pulsed dye laser versus long pulsed Nd:YAG laser treatment in recalcitrant viral warts". J Dermatol Treat 2017;28(5): 411-416

4. El-Mohamady A, Mearag I, El-khalawany M, Elshahed A, et al. "Pulsed dye laser versus Nd:YAG laser in the treatment of plantar warts: a comparative study". Lasers Med Sci. 2014; 29:1111-1116

5. Veitch D, Kravvas G, Al-niaimi F. "Pulsed Dye Laser Therapy in the Treatment of Warts: A Review of the Literature". Dermatol Surg 2017; 43:485-493 